\title{
The subepicardial microcirculation in heterotopically transplanted mouse hearts: An intravital multifluorescence microscopy study
}

René Schramm, MD, PhD, ${ }^{\mathrm{a}, \mathrm{b}}$ Michael D. Menger, MD, ${ }^{\mathrm{b}}$ Sarah Kirsch, ${ }^{\mathrm{a}, \mathrm{b}}$ Frank Langer, MD, ${ }^{\mathrm{a}}$ Yves Harder, MD, Jürg Hamacher, MD, ${ }^{\mathrm{c}}$ and Hans-Joachim Schäfers, MD $^{\mathrm{a}}$

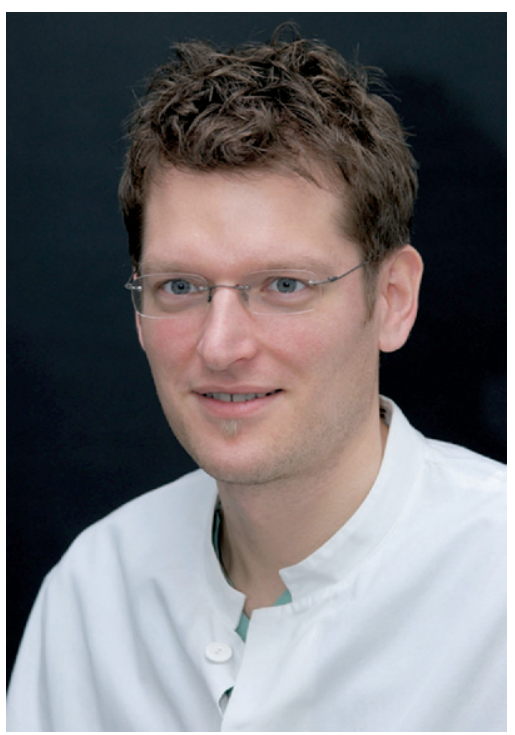

Dr Schramm

Supplemental material is available online.
From the Departments of Thoracic and Cardiovascular Surgery ${ }^{\mathrm{a}}$ and Internal Medicine $\mathrm{V},{ }^{\mathrm{c}}$ and the Institute for Clinical-Experimental Surgery, ${ }^{\mathrm{b}}$ University of Saarland, Homburg/Saar, Germany.

Received for publication July 27, 2006; revisions received Jan 9, 2007; accepted for publication Feb 12, 2007.

Address for reprints: René Schramm, MD, $\mathrm{PhD}$, Department of Thoracic and Cardiovascular Surgery, Institute for ClinicalExperimental Surgery, University of Saarland, D-66421 Homburg/Saar, Germany (e-mail: reneschramm@hotmail.com).

J Thorac Cardiovasc Surg 2007;134:210-7

$0022-5223 / \$ 32.00$

Copyright () 2007 by The American Association for Thoracic Surgery

doi:10.1016/j.jtcvs.2007.02.018
Objective: We developed a model for intravital microscopic analysis of the coronary microcirculation in transplanted murine hearts and assessed the influence of cold ischemia on postischemic microcirculatory dysfunctions.

Methods: Murine hearts were exposed to $60(\mathrm{n}=12)$ and 240 minutes $(\mathrm{n}=8)$ of cold ischemia before syngeneic heterotopic transplantation. Intravital fluorescence microscopy allowed detailed analysis of the right ventricular coronary microcirculation, including feeding arterioles, nutritive capillaries, and postcapillary venules. With this technique, we further studied leukocyte-endothelial cell interactions, microvascular permeability, tissue oxygenation, and microlymphatics.

Results: Cold ischemia of 240 minutes aggravated nutritive capillary perfusion failure, indicated by a significant reduction of functional capillary density and capillary flow velocity by $63 \%$ and $45 \%(P<.05$ vs 60 -minute cold ischemic isografts). The mitochondrial redox state, visualized by nicotinamide adenine dinucleotide hydrogen autofluorescence, was markedly deteriorated after 240-minute cold ischemia $(P<.05)$, indicating a persistent mismatch between oxygen supply and demand resulting from pronounced capillary no-reflow. Prolonged ischemia further resulted in 6- and 11-fold higher numbers of rolling and firmly adherent leukocytes in postcapillary venules $(P<.05)$, together with increased microvascular permeability.

Conclusions: We introduce a novel approach to visualize in detail the murine coronary microcirculation in vivo by multifluorescence microscopy. Our data demonstrate that prolonged cold ischemia provokes posttransplant capillary no-reflow, leukocytic inflammation, and persistent tissue hypoxia.

$\mathrm{M}$ yocardial ischemia/reperfusion (I/R) injury is associated with cardiomyocyte necrosis, arrhythmia, low cardiac output, and myocardial stunning. The pathophysiologic mechanisms involve the production of oxygenderived free radicals, activation of the complement system, calcium overload with subsequent hypercontracture, platelet deposition and microthrombus formation, capillary no-reflow and endothelial dysfunction, polymorphonuclear leukocyte sequestration, and, finally, loss of cellular integrity. ${ }^{1-3}$ It is still a matter of debate, however, whether I/R-related myocardial accumulation of neutrophils ultimately corresponds to postischemic cardiac dysfunction. ${ }^{4}$ The overt discrepancies between research data most likely relate to the fact that the detailed molecular and microvascular mechanisms that support leukocytic recruitment in the myocardium are not completely understood.

Numerous experimental studies have utilized intravital microscopy to directly visualize the microvascular alterations and the migratory events supporting tissue sequestration of leukocytes during I/R injury in various organs. ${ }^{\text {E1-E5 }}$ Such observa- 


\section{Abbreviations and Acronyms \\ FCD = functional capillary density \\ FITC = fluorescein isiothiocyanate \\ $\mathrm{I} / \mathrm{R}=$ ischemia/reperfusion \\ LAD $=$ left anterior descending coronary artery \\ $\mathrm{NADH}=$ nicotinamide adenine dinucleotide hydrogen \\ $\mathrm{RBC}=$ red blood cell \\ SEM $=$ standard error of the mean}

tions have been limited in the heart because (1) the animals require mechanical ventilation after thoracotomy, and (2) the movements of both the heart and the lung hinder continuous in vivo high-resolution microscopic imaging of the coronary microcirculation. In mice, intravital microscopic analysis of the coronary microcirculation has not been described. This is in contrast to the growing interest in experimental mouse models due to the availability of a huge array of specific monoclonal antibodies directed against murine epitopes and genetically modified animals. Data on murine myocardial I/R injury have frequently been gathered indirectly from ex vivo sanguineous perfusion experiments of the isolated mouse heart or by extrapolating data obtained from intravital microscopic studies of striated muscle preparations. $^{5-7}$

Herein, we introduce a novel experimental model that allows assessment of the murine coronary microcirculation by high-resolution intravital multifluorescence microscopy. With this approach, we studied the effect of cold ischemia time on coronary microcirculatory dysfunction, myocardial tissue reoxygenation, and leukocyte-endothelial cell interactions during the postischemic reperfusion of heterotopically transplanted murine cardiac isografts.

\section{Materials and Methods \\ Animals}

Inbred C57BL/6 mice with a body weight ranging between 26 and $28 \mathrm{~g}$ were used as donors and recipients. Animals were housed in a 12-hour dark-light cycle and were allowed free access to standard pellet food (Altromin, Lage, Germany) and tap water ad libitum. All experiments were performed in accordance with the legislation on the protection of animals and were approved by the Governmental Ethical Committee for Animal Experimentation. The investigation conforms to the Guide for Care and Use of Laboratory Animals published by the US National Institutes of Health (NIH Publication No. 85-23, revised 1996). For all surgical interventions, animals were anesthetized by an intraperitoneal injection of $7.5 \mathrm{mg}$ ketamine hydrochloride (Parke Davis, Freiburg, Germany) and $2.5 \mathrm{mg}$ xylazine (Bayer, Leverkusen, Germany) per $100 \mathrm{~g}$ body weight and were placed on a heating pad to maintain a body temperature of $37^{\circ} \mathrm{C}$ throughout the entire experimental procedures. The anesthetized animals were killed after experimentation by midline sternotomy, diaphragmatomy, and ventriculotomy.

\section{Heterotopic Heart Transplantation}

Murine cardiac syngrafts were transplanted heterotopically to the recipients' cervical vessels, as described previously. ${ }^{8}$ Briefly, cold ischemia was induced by incising the donor subrenal vena cava and concomitant retrograde infusion of ice-cold phosphatebuffered saline solution into the abdominal aorta (10 minutes, 100 $\mathrm{mm} \mathrm{H}_{2} \mathrm{O}$ ). The grafts were quickly harvested and stored on ice for either 60 or 240 minutes before heterotopic transplantation. All transplantations were performed by 1 trained microsurgeon. The success rate of the operative procedure was $\sim 90 \%$, as indicated by vital and functioning grafts after recipient survival of the surgical procedure. In the 60-minute cold ischemia group, 14 animals received heterotopic heart transplantation. Twelve animals survived the surgical procedure without complications and could be included in the study group. Two animals showed bleeding from the arterial anastomosis after removal of the vascular clamps. In the 240-minute cold ischemia group, 9 animals received heterotopic heart transplantation. Eight animals survived the surgical procedure without complications and could be included in the study group. One animal showed bleeding from the arterial anastomosis after removal of the vascular clamps. Revision of the anastomosis would have caused drastic prolongation of the warm ischemia time, and thus the 3 animals with bleeding after the surgical procedure were excluded from the study.

\section{Intravital Fluorescence Microscopy}

After transplantation, the animals were transferred to the intravital microscope stage, and the upper surface of the right ventricular myocardium was covered by a glass slip. To avoid compression of the tissue and blood vessels, the glass slip was adjusted by a micromanipulator so that the epicardial surface, which was moistened by phosphate-buffered saline solution, adhered to the lower surface of the glass slip. The coronary microvasculature and myocardial surface were visualized by means of a modified Zeiss Axiotech microscope (Zeiss, Oberkochen, Germany) equipped with a 100-W mercury lamp and filter sets for blue (450-490-nm excitation and $>520-\mathrm{nm}$ emission wavelength), green (530-560 $\mathrm{nm} ;>580 \mathrm{~nm})$, and near-ultraviolet $(330-390 \mathrm{~nm} ;>430 \mathrm{~nm})$ light epi-illumination. Microscopic images were televised and recorded by means of a charge-coupled device video camera (FK6990, Pieper, Schwerte, Germany) and recorded on videotape (Panasonic AG-7350-S-VHS, Matsushita, Tokyo, Japan) for subsequent offline analyses of microcirculatory parameters utilizing the CapImage Software (Zeintl, Heidelberg, Germany).

The nicotinamide adenine dinucleotide hydrogen (NADH) autofluorescence, which reflects the mitochondrial redox state and serves as a measure for tissue oxygenation, ${ }^{9}$ was assessed by defined near-ultraviolet light epi-illumination of the ischemic isografts prior to and at 20,40, and 60 minutes of reperfusion.

Leukocyte-endothelial cell interactions were analyzed at 60 minutes of reperfusion by in vivo labeling of circulating leukocytes with Rhodamine 6G (0.01 mg intravenously, Sigma Chemical Co., St. Louis, Mo) and green light epi-illumination. Leukocytic rolling was determined by counting the number of leukocytes that were rolling along the endothelial microvascular surface and passing a reference point during 30 seconds, expressed as cells $\cdot \min ^{-1} \cdot \mathrm{mm}^{-1}$ microvascular circumference. Leukocytic firm adhesion was determined by counting the number of Rhoda- 

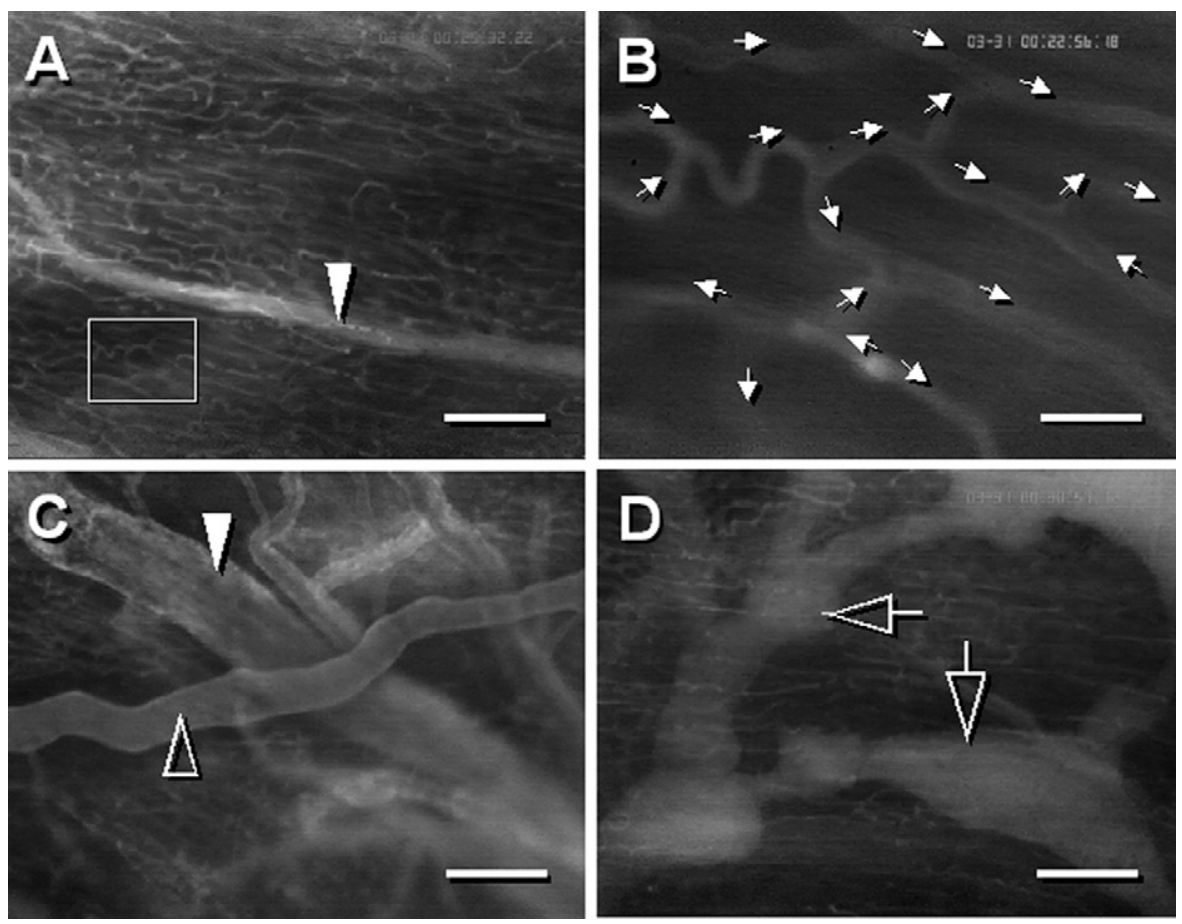

Figure 1. Intravital fluorescence microscopy of the subepicardial microcirculation in syngeneically transplanted mouse hearts. The intravital fluorescence microscopy micrographs show the coronary network of nutritive capillaries (A), individual postcapillary venules (A and $C$, solid arrow heads), arterioles (C, open arrow head), and intramyocardial lymphatic ducts (D, open arrows) in a 60-minute cold ischemic graft. The insert of $(A)$ is given in higher magnification in (B), demonstrating the mixed cocurrent and countercurrent capillary flow pattern ( $B$, filled arrows indicate the direction of capillary blood flow). Contrast enhancement was achieved by intravenous injection of FITC-dextran 150,000. The scale bars represent 100 $\mu \mathrm{m}(\mathrm{A}, \mathrm{C}, \mathrm{D})$ and $20 \mu \mathrm{m}(\mathrm{B})$. mine 6G-labeled leukocytes remaining stationary at the endothelial surface within a coronary microvascular segment for at least 30 seconds, expressed in cells $/ \mathrm{mm}^{2}$ endothelial surface. Contrast enhancement with fluorescein isiothiocyanate (FITC)-dextran (molecular weight 150,000, $0.1 \mathrm{mg}$ intravenously; Sigma Chemical Co.) and blue light epi-illumination were used to determine functional capillary density (FCD), which is the length of perfused nutritive capillaries per area of subepicardial capillary network expressed in $\mathrm{cm} / \mathrm{cm}^{2}$. Nutritive capillary diameters were expressed in micrometers. Capillary and venular red blood cell (RBC) flow velocities were expressed in $\mathrm{mm} / \mathrm{s}$. The distinct fluorescence intensities of FITC-dextran within postcapillary coronary venules and adjacent extravascular tissue compartments were measured densitometrically. The macromolecular leakage (microvascular permeability) was then calculated as the ratio between the extraand intravascular FITC-dextran fluorescence intensity. All microcirculatory parameters were measured in 3 to 5 different areas of the subepicardial coronary microcirculation in all transplanted hearts.

\section{Survival of Cardiac Syngrafts}

In 4 separate experiments, syngeneic transplantations were performed without subsequent intravital microscopy. In contrast, following release of the microvascular hemostats and after beginning of reperfusion, the neck skin was closed by a continuous suture (5-0 polyethylene), and the animals were allowed to recover from anesthesia. The syngraft function was controlled daily during the following 120 days by inspection and simple palpation of the beating graft under the anterior neck skin. After the observation period, the animals were anesthetized and killed as described above. These experiments were meant to demonstrate that the transplantation procedures were successfully performed without vascular thrombosis and associated dysfunction and to show that cardiac syngrafts survive indefinite solely exposed to I/R injury without acute or chronic immune rejection. No immunosuppression was used.

\section{Statistics}

Data are given as mean values \pm standard error of the mean (SEM) and $n$ represents the number of animals per group. The coefficient of variance (i.e., the ratio between standard deviation and mean value) was calculated to assess the intraindividual heterogeneity of microcirculatory parameters. Statistical differences were calculated using the Mann-Whitney rank sum test (Sigma Stat Software, Jandel Scientific, San Rafael, Calif).

\section{Results}

The syngeneically transplanted murine hearts were reperfused directly after removal of the vascular hemostats changing from their faint grayish color to a vivid red aspect. The syngrafts started beating in a stable rhythm within 2 minutes of reperfusion, as was also reported previously by others. ${ }^{8}$ In animals that were allowed to recover from anesthesia, the syngeneically transplanted hearts performed well and pulsated under the ventral neck skin throughout the entire 120-day postoperative course, independent of the cold ischemia time.

Intravital fluorescence microscopy allowed detailed imaging of the subepicardial microcirculation of the beating grafts during postischemic reperfusion. This included myocardial nutritive capillaries and draining postcapillary venules (Figure 1, $A$ and $B$ ). The flow pattern of neighboring 

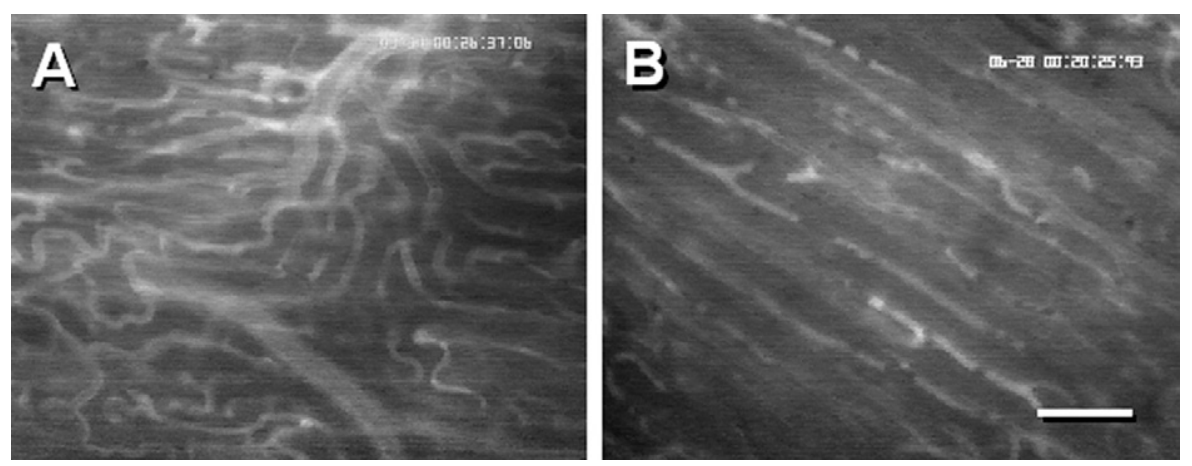

Figure 2. Postischemic perfusion failure of nutritive myocardial capillaries of murine cardiac isografts. The intravital fluorescence microscopy micrographs show the capillary network of the subepicardial myocardium of a 60 minute (A) and a 240-minute cold ischemic graft (B) after 60 minutes of reperfusion. Contrast enhancement was achieved by intravenous injection of FITCdextran 150,000. Scale bar represents $40 \mu \mathrm{m}$.

capillaries was found to be mainly mixed cocurrently and countercurrently with abundant cross-connections (Figure 1, A and $B$ ). Coronary arterioles could be visualized close to the atrioventricular transition (Figure 1,C). In general, arterial microvessels descend into the myocardium early in their proximal course and proceed intramurally at more distal sites, where the accessibility for epi-illumination intravital microscopy is restricted. In addition to blood vessels, dilated lymph vessels could be found, gleaming from the deep myocardium (Figure 1,D).

The time of cold ischemic storage had great impact on the magnitude of myocardial perfusion failure during postischemic reperfusion of the isografts (Figure 2). This was indicated by a significantly lower FCD and capillary RBC flow velocity in 240-minute cold ischemic grafts when compared with those stored for only 60 minutes (Table 1). The coefficient of variance of the capillary RBC velocity was comparable and low in both groups, indicating a low intraindividual heterogeneity of the capillary RBC flow velocity in both groups (Table 1). The coefficient of variance of the FCD, however, was significantly higher after 240-minute cold storage, indicating a higher intraindividual heterogeneity of the myocardial capillary perfusion after prolonged ischemia (Table 1). The mean diameter of reperfused capillaries ranged between 4 and $8 \mu \mathrm{m}$ and was comparable between 60- and 240-minute cold ischemic grafts (data not shown).

In postcapillary venules, we also found a marked reduction of the venular RBC flow velocity after 240 minutes versus 60 minutes of cold storage (Table 1). The corresponding coefficient of variance indicated a low intraindividual heterogeneity of the venular RBC flow velocity without significant differences between the groups (Table 1).

Analysis of microvascular permeability (macromolecular leakage) revealed increased values after 240 minutes of cold storage when compared with those measured after 60 minutes of storage, although this difference did not prove to be statistically significant (Table 1). The coefficient of variance of the macromolecular leakage indicated a homogenous intraindividual endothelial dysfunction pattern in both groups (Table 1).

The intensity of the ischemia-induced NADH autofluorescence declined during the first 60 minutes of reperfusion in both groups $(P<.05$ vs 0 min of reperfusion; Figure 3$)$. However, after 60-minute cold storage, the peak level of NADH autofluorescence dropped to approximately $50 \%$ during the first hour of reperfusion, and 240-minute stored

TABLE 1. Microcirculatory parameters

\begin{tabular}{lcc}
\hline & \multicolumn{2}{c}{ Cold ischemia time } \\
\cline { 2 - 3 } & $\begin{array}{c}\mathbf{6 0} \mathbf{~ m i n} \\
(\mathbf{n}=\mathbf{1 2})\end{array}$ & $\begin{array}{c}\mathbf{2 4 0} \mathbf{m i n} \\
(\mathbf{n}=\mathbf{8})\end{array}$ \\
\hline Microvascular perfusion & & \\
FCD (cm/cm ${ }^{2}$ ) & $840 \pm 57$ & $312 \pm 35^{*}$ \\
$\quad$ Coefficient of variance & $0.14 \pm 0.04$ & $0.52 \pm 0.09^{*}$ \\
Capillary RBC flow velocity (mm/s) & $0.36 \pm 0.04$ & $0.20 \pm 0.05^{*}$ \\
$\quad$ Coefficient of variance & $0.34 \pm 0.07$ & $0.29 \pm 0.09$ \\
Venular RBC flow velocity (mm/s) & $0.78 \pm 0.07$ & $0.51 \pm 0.10^{*}$ \\
$\quad$ Coefficient of variance & $0.44 \pm 0.08$ & $0.47 \pm 0.11$ \\
Inflammation & & \\
Microvascular permeability (\%) & $0.57 \pm 0.02$ & $0.73 \pm 0.09^{*}$ \\
Coefficient of variance & $0.12 \pm 0.02$ & $0.17 \pm 0.04$ \\
Leukocytic rolling (cells $\cdot$ min $^{-1}$. & $33 \pm 12$ & $201 \pm 52^{*}$ \\
$\quad$ mm $^{-1}$ ) & & \\
Coefficient of variance & $1.21 \pm 0.11$ & $1.09 \pm 0.15$ \\
Leukocytic firm adhesion (cells/ & $58 \pm 21$ & $630 \pm 132^{*}$ \\
mm $^{2}$ ) & & \\
Coefficient of variance & $1.06 \pm 0.13$ & $1.06 \pm 0.12$
\end{tabular}

The subepicardial microcirculation was assessed by intravital multifluorescence microscopy. Parameters assessing microvascular perfusion and inflammation were determined by computer-assisted off-line analysis of recorded images. The coefficient of variance was calculated intraindividually and is defined as the ratio between standard deviation and the mean value. Data are given as mean values \pm SEM. Twelve and 8 experiments were performed and analyzed to assess the effects of 60 and 240 minutes of cold ischemia, respectively, on postischemic microvascular dysfunction. ${ }^{*} P<.05$ versus 60 -minute cold ischemic grafts. FCD, Functional capillary density; $R B C$, red blood cells. 

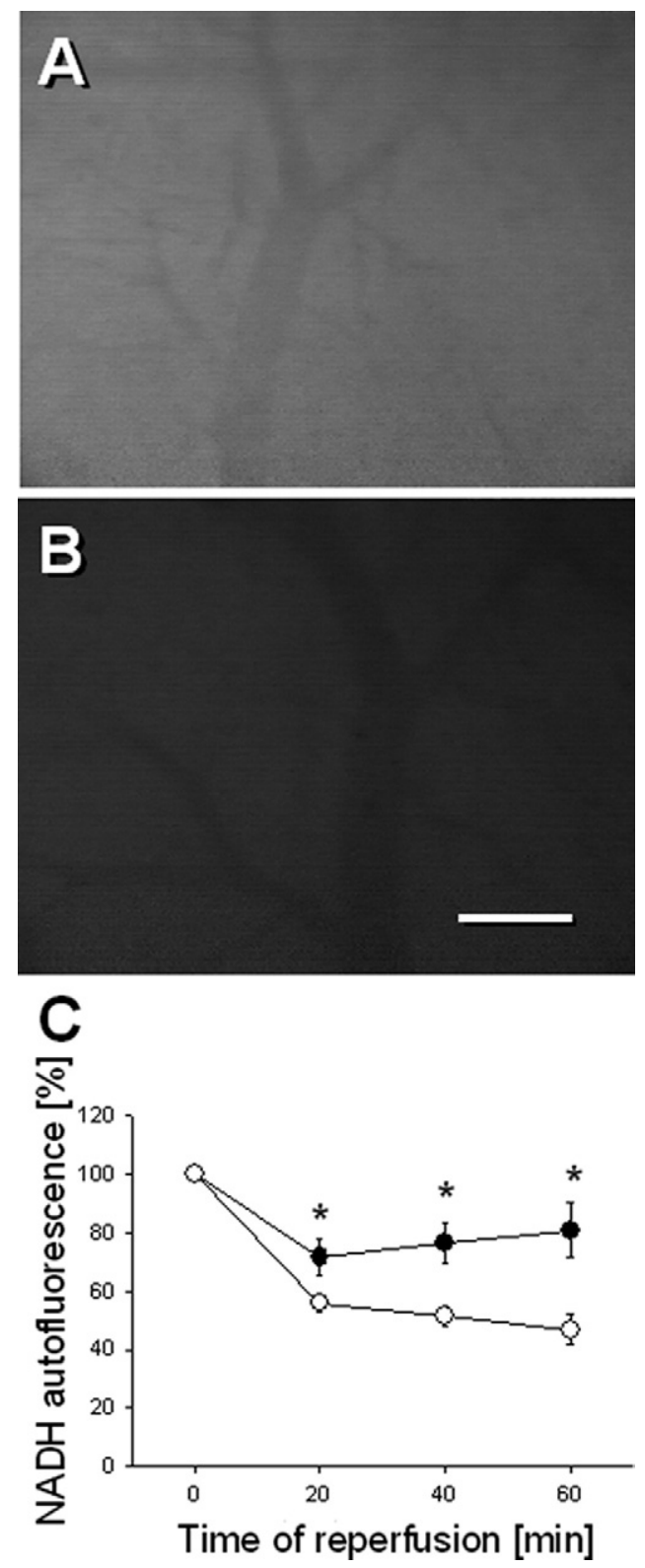

Figure 3. NADH autofluorescence of murine cardiac isografts. The intravital fluorescence microscopy micrographs show the identical area of the right ventricular subepicardial myocardium in a 60-minute cold ischemic isograft just before $(A)$ and at 40 minutes after onset of reperfusion (B). Scale bar represents 100 $\mu \mathrm{m}$. C, The quantitative assessment of the NADH autofluorescence of isografts that underwent 60 minutes (white circles) and 240 minutes (black circles) of cold storage just before $(0 \mathrm{~min}$ ) as well as at 20, 40, and 60 minutes of reperfusion. Data are expressed in percent of the peak values measured at $\mathbf{0}$ minutes of reperfusion and are given as mean \pm SEM. ${ }^{*} P<.05$ versus 60 -minute cold storage; $n=12$ and $n=8$ experiments were performed and analyzed to assess the effects of 60 and 240 minutes of cold ischemia, respectively. grafts showed an only $25 \%$ reduction of NADH autofluorescence during postischemic reperfusion $(P<.05$, Figure 3$)$.

The magnitude of the leukocytic response within the postischemic myocardium was dependent on the duration of cold ischemic preservation. Leukocytic rolling and leukocytic firm adhesion were only occasionally observed in reperfused postcapillary venules of the 60-minute cold ischemic grafts (Figure 4, B). In contrast, the 240-minute cold ischemic grafts presented with markedly enhanced venular leukocytic sequestration (Figure 4,D), including a sixfold increase in leukocytic rolling and an 11-fold increase in leukocytic firm adhesion $(P<.05$; Table 1$)$. The coefficient of variance of leukocytic rolling and leukocytic firm adhesion indicated a remarkable heterogeneous intraindividual distribution pattern of leukocyte-endothelial cell adhesive interactions in postcapillary coronary venules in both groups.

Of note, virtually no leukocyte-endothelial cell interactions were found in coronary arterioles, independent of the different cold ischemia times applied.

\section{Discussion}

We herein analyzed for the first time the murine coronary microcirculation in vivo by imaging the entire microvascular network using epi-illumination multifluorescence microscopy. Although previous investigations have utilized specially designed heart-holding devices to study coronary microvascular responses, ${ }^{10-13}$ we used the heterotopic cervical heart transplantation as a basic model for free access to the murine mouse heart. The extrathoracic localization of the transplanted graft excludes the need for invasive ventilation and counterbalance of respiratory movements. This is due to the fact that the grafts do not perform normal contractions. The grafts experience a reduced diastolic filling and thus only mild systolic ejection of almost exclusively the right heart. Correspondingly, the right atrium and ventricle remain in a working state. The left atrium and ventricle are in a nonworking state and experience virtually no filling. They even undergo significant atrophy during chronic experiments. ${ }^{14}$

The heterotopic heart transplantation model seems advantageous over classical left anterior descending coronary artery (LAD) occlusion experiments when studying myocardial I/R injury. ${ }^{15}$ After syngeneic transplantation, the entire myocardium of the graft is equally exposed to defined $\mathrm{I} / \mathrm{R}$ injury, and the infarcted region of the myocardium after occlusion of the LAD is considerably variable in size and mainly distributed to the subendocardial myocardium, which is not accessible for intravital epi-illumination microscopy. ${ }^{16}$

The heterotopic heart transplantation model, however, may appear less appropriate to study the murine myocardial microcirculation under other than postischemic conditions. 

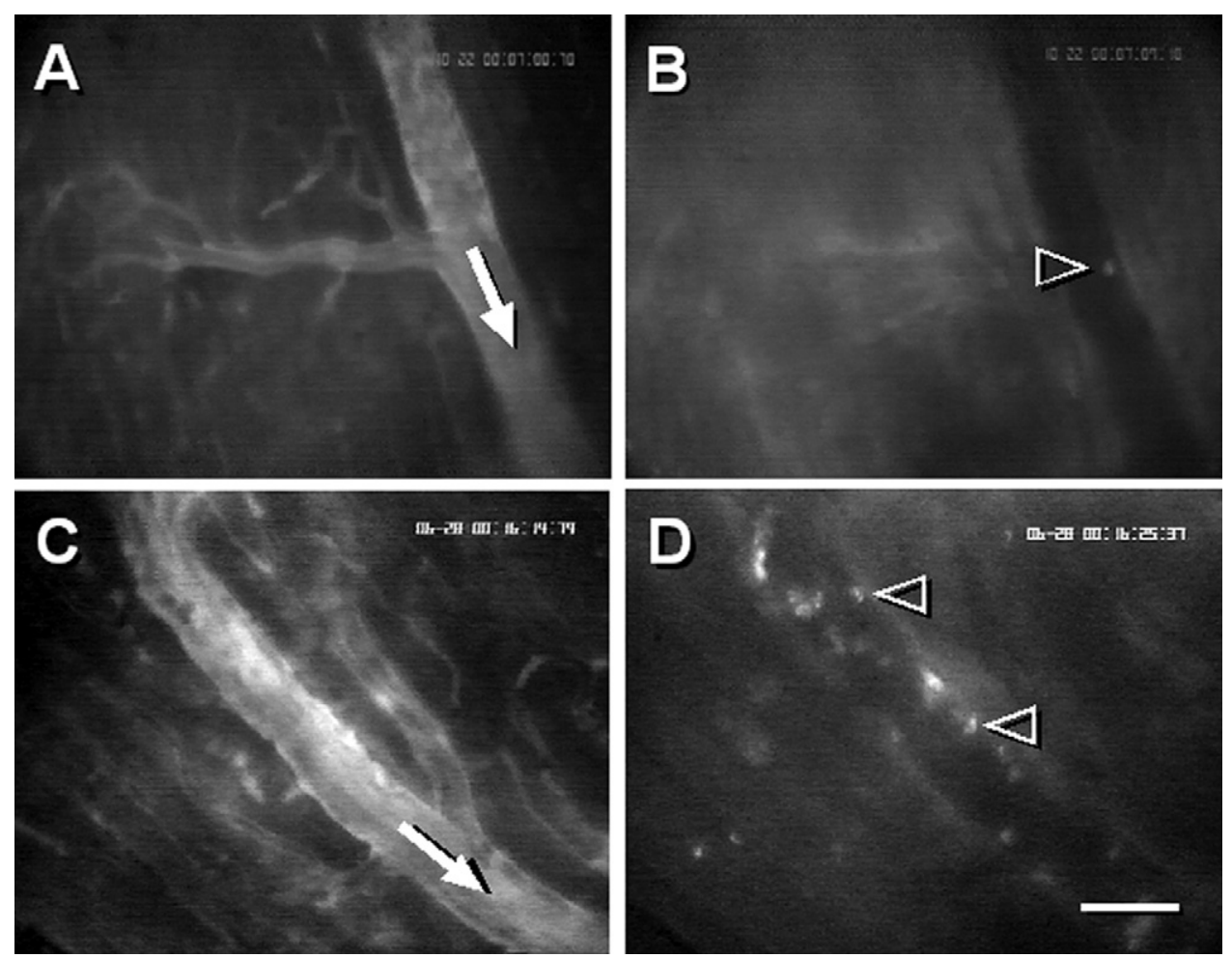

Figure 4. Leukocyte-endothelial cell adhesive interactions in the coronary microcirculation of murine cardiac syngrafts. A and B, One postcapillary coronary venule of the subepicardium of a 60 -minute cold ischemic syngraft at 60 minutes of posttransplant reperfusion. $C$ and $D, 0$ ne postcapillary coronary venule of the subepicardium of a 240-minute cold ischemic syngraft at 60 minutes of posttransplant reperfusion. The micrographs in (A) and (C) were generated by using blue light epi-illumination, allowing precisely delineation of the structure of the venule due to intravascular FITC-dextran 150,000. The micrographs in (B) and (D) were generated by using green light epi-illumination, allowing visualization of leukocyte-endothelial cell adhesive interaction due to intracellular staining with Rhodamin 6G. The direction of blood flow is indicated by solid arrows (A and C). Leukocytes interacting with the venular endothelium are indicated by open arrowheads (B and D). The scale bar represents approximately $40 \mu \mathrm{m}$.

Those experiments should be performed after a 2- to 3-day recovery from the ischemia-associated transplantation procedure. This, of course, requires minimization of the graft total ischemia time, which can be achieved by using optimized organ cold preservation and procurement strategies. ${ }^{\text {E6,17 }}$ Accordingly, others have frequently used the model of heterotopic heart transplantation in mice to study various disease states, including the recipients adaptive immune reactions after allogeneic heart transplantation. ${ }^{18}$ Performing allogeneic transplantation, our presented model not only may serve for studying the role of initial $I / R$ injury for the manifestation of later rejection but also may be used to study the coronary microcirculation during posttransplant acute and chronic rejection. Further, the model may allow for studying the effects of distinct preservation strategies, brain death, and certain immunosuppressive therapy regimens on the postischemic myocardial microcirculation.
The blood flow pattern in the myocardial capillaries was found to be mixed cocurrently and countercurrently with abundant cross-connections, which is in line with previous findings in native hearts of dogs and rats. ${ }^{13,19}$ Our data show prolonged myocardial ischemia caused marked capillary perfusion failure during myocardial reperfusion. A recruitment of a functional capillary reserve was not found when comparing 2 different cold ischemia times. Previous reports on hypoxia-induced coronary microvascular alterations could also not confirm myocardial capillary recruitment in the native rat and dog heart. ${ }^{19-22}$ In fact, a coronary capillary flow reserve with increased capillary blood flow via capillary cross-connections and capillary dilation was only described during reactive hyperemia after a 20 -second shortterm occlusion of the LAD. ${ }^{19}$ The lack of recruitment of capillary perfusion indicates that the heterotopically transplanted mouse hearts experienced significant injury after 
both 60 minutes and 240 minutes of cold ischemia. The I/R injury-associated capillary no-reflow may simply have overshadowed any eventual capillary recruitment by activation of the capillary flow reserve.

Measurement of NADH autofluorescence has frequently been used to determine the mitochondrial redox state and, thus, tissue oxygenation. Increased NADH autofluorescence indicates tissue hypoxia, and improvement of tissue oxygenation is associated with a decrease of NADH fluorescence intensity. Although this technique has already been applied in liver and striated muscle in vivo, ${ }^{9}$ in myocardial tissue it has been used only in cell culture and isolated hearts. ${ }^{23,24}$ Herein, we have applied this technique for the first time to study oxygenation of myocardial tissue in vivo and could demonstrate that the NADH autofluorescence declines during the first hour of reperfusion. This indicates successful reoxygenation of the myocardial tissue and recovery from the ischemic insult. However, myocardial reoxygenation was less pronounced in isografts that underwent prolonged cold storage. This displays a persistently higher mismatch between myocardial oxygen supply and demand in those hearts exposed to prolonged ischemia, which is presumably due to the augmented capillary noreflow. Of note, the reduction of NADH autofluorescence in the 60-minute cold ischemic grafts may not necessarily illustrate a complete restoration of myocardial oxygen supply, because our experimental setup does not permit a comparison with baseline measurements of undisturbed native murine myocardium.

Vascular endothelial dysfunction is an additional characteristic of I/R injury. The properties of deterioration of endothelium-regulated vascular function include flow, permeability, and cell trafficking. ${ }^{25}$ Because it is evident that edema of the myocardium contributes to postischemic cardiac dysfunction, ${ }^{26}$ we analyzed herein the postischemic myocardial edema formation by determining the extravasation of the intravenously applied high-molecular-weight FITC-dextran. Our data suggest significant disruption of the endothelial barrier function in the postischemic myocardial microcirculation, considering that a ratio between extra- and intravascular FITC-dextran fluorescence intensity of $>0.5$ represents a pathologic increase of endothelial permeability in tissues with nonfenestrated endothelium. ${ }^{27}$ Although we found that the macromolecular leakage was higher in hearts exposed to prolonged ischemia, the difference between the groups did not prove to be statistically significant. However, we assessed the microvascular leakage only at 1 hour after onset of reperfusion and, thus, cannot exclude that prolonged ischemia may exert a more pronounced endothelial disintegration in the later time course of reperfusion.

The accumulation of neutrophils has been recognized as a hallmark of myocardial infarction for more than 60 years. $^{2}$
However, there is still debate on the impact of neutrophil sequestration on myocardial I/R injury, infarction size, and postischemic cardiac dysfunction. ${ }^{4}$ Several adhesion molecules known to support postischemic leukocyte-endothelial cell interactions in other tissues are also expressed in the myocardium, indicating a specific regulation of myocardial leukocytic retention during $\mathrm{I} / \mathrm{R}$ injury. ${ }^{28-30}$ Nonetheless, little is known about the functional significance of the distinct adhesion receptors in supporting the individual steps of the leukocytic extravasation cascade in the heart (e.g. rolling, tethering, firm adhesion, and transendothelial migration). The lack of such detailed knowledge may also explain why therapeutic strategies targeting single adhesion receptors failed to improve the outcome after myocardial infarction. ${ }^{\mathrm{E}, \mathrm{E} 8}$ Our novel approach described herein provides sufficient temporal and spatial resolution to study leukocyte-endothelial cell interactions in the murine coronary microcirculation in detail. The data show that intravial microscopy of the subepicardial microcirculation in heterotopically transplanted murine cardiac isografts permits differentiated analysis of leukocytic rolling and firm adhesion in coronary microvessels. Although a previous study has reported that the postischemic myocardial no-reflow may be due to leukocytic plugging in myocardial capillaries, ${ }^{31}$ we found that coronary postcapillary venules are the predominant site for postischemic myocardial leukocytic sequestration. Both leukocytic rolling and firm adhesion were significantly elevated in postcapillary coronary venules after prolongation of the cold ischemia time, which was associated with a marked reduction of the postcapillary venular blood flow velocity. Future studies have to clarify whether the augmented venular leukocytic sequestration in the postischemic myocardium is simply due to changes in coronary blood flow properties or based on increased expression and specific function of distinct adhesion molecules.

Our study demonstrates that intravital fluorescence microscopy of heterotopically transplanted mouse hearts enables for detailed imaging of the murine subepicardial coronary microcirculation in vivo. This novel approach has been proven to be accurate and reproducible to study effects of myocardial I/R on the coronary microcirculation. Our data demonstrate that prolonged periods of ischemia deteriorate the nutritive myocardial perfusion and enhance leukocytic inflammation during postischemic reperfusion. Moreover, the novel experimental setup may also be used to study the coronary microcirculation during cardiac transplantation pathology. ${ }^{17,20,21,22,28,29,30}$

\section{References}

1. Moens AL, Claeys MJ, Timmermans JP, Vrints CJ. Myocardial ischemia/reperfusion-injury, a clinical view on a complex pathophysiological process. Int J Cardiol. 2005;100:179-90.

2. Mallory GK, White PD. The speed of healing of myocardial infarction. Am Heart J. 1939;18:647-71. 
3. Kloner RA, Ganote CE, Jennings RB. The "no-reflow" phenomenon after temporary coronary occlusion in the dog. J Clin Invest. 1974;54: 1496-508.

4. Baxter GF. The neutrophil as a mediator of myocardial ischemiareperfusion injury: time to move on. Basic Res Cardiol. 2002;97: 268-75.

5. Kupatt C, Habazettl H, Zahler S, Weber C, Becker BF, Messmer K, et al. ACE-inhibition prevents postischemic coronary leukocyte adhesion and leukocyte-dependent reperfusion injury. Cardiovasc Res. 1997;36: 386-95.

6. Goebel S, Kuebler WM, Cornelissen AJ, Kuppe H, Pries AR, Habazettl $\mathrm{H}$. In situ analysis of coronary terminal arteriole diameter responses: technical report of a new experimental model. J Vasc Res. 2003;40:442-8.

7. Johns DG, Ao Z, Willette RN, Macphee CH, Douglas SA. Role of p38 MAP kinase in postcapillary venule leukocyte adhesion induced by ischemia/reperfusion injury. Pharmacol Res. 2005;51:463-71.

8. Matsuura A, Abe T, Yasuura K. Simplified mouse cervical heart transplantation using a cuff technique. Transplantation. 1991;51: 896-8.

9. Vollmar B, Burkhardt M, Minor T, Klauke H, Menger MD. Highresolution microscopic determination of hepatic NADH fluorescence for in vivo monitoring of tissue oxygenation during hemorrhagic shock and resuscitation. Microvasc Res. 1997;54:164-73.

10. Vollmar B, Conzen P, Habazettl H, Adili F, Peter K. Does nitrous oxide affect coronary microcirculation? An intravital microscopic study in the canine heart. Anesth Analg. 1995;80:249-55.

11. Jones CJ, DeFily DV, Patterson JL, Chilian WM. Endotheliumdependent relaxation competes with alpha 1- and alpha 2-adrenergic constriction in the canine epicardial coronary microcirculation. $\mathrm{Circu}$ lation. 1993;87:1264-74.

12. Huwer H, Rissland J, Vollmar B, Nikoloudakis N, Welter C, Menger $\mathrm{MD}$, et al. Angiogenesis and microvascularization after cryothermiainduced myocardial infarction: a quantitative fluorescence microscopic study in rats. Basic Res Cardiol. 1999;94:85-93.

13. Steinhausen M, Tillmanns H, Thederan H. Microcirculation of the epimyocardial layer of the heart. I. A method for in vivo observation of the microcirculation of superficial ventricular myocardium of the heart and capillary flow pattern under normal and hypoxic conditions. Pflugers Arch. 1978;378:9-14.

14. Heron I. A technique for accessory cervical heart transplantation in rabbits and rats. Acta Pathol Microbiol Scand [A]. 1971;79:366-72.

15. Fishbein MC, Maclean D, Maroko PR. Experimental myocardial infarction in the rat: qualitative and quantitative changes during pathologic evolution. Am J Pathol. 1978;90:57-70.

16. Samsamshariat SA, Samsamshariat ZA, Movahed MR. A novel method for safe and accurate left anterior descending coronary artery ligation for research in rats. Cardiovasc Revasc Med. 2005;6:121-3.
17. Schramm R, Schafers HJ, Hamacher J, Menger MD. Simplified technique for heterotopic vascularized heart transplantation in mice. $\mathrm{Mi}$ crosurgery. 2006;26:212-3.

18. Corbascio M, Ekstrand H, Osterholm C, Qi Z, Simanaitis M, Larsen $\mathrm{CP}$, et al. CTLA4Ig combined with anti-LFA-1 prolongs cardiac allograft survival indefinitely. Transpl Immunol. 2002;10:55-61.

19. Kiyooka T, Hiramatsu O, Shigeto F, Nakamoto H, Tachibana H, Yada $\mathrm{T}$, et al. Direct observation of epicardial coronary capillary hemodynamics during reactive hyperemia and during adenosine administration by intravital video microscopy. Am J Physiol Heart Circ Physiol. 2005;288:H1437-43.

20. Steinhausen M, Tillmanns H, Thederan H, Leinberger H. Microcirculation of the epimyocardial layer of the rat heart: no recruitment after hypoxia. Bibl Anat. 1979;18:85-9.

21. Ashikawa K, Kanatsuka H, Suzuki T, Takishima T. Phasic blood flow velocity pattern in epimyocardial microvessels in the beating canine left ventricle. Circ Res. 1986;59:704-11.

22. Tillmanns H, Ikeda S, Hansen H, Sarma JS, Fauvel JM, Bing RJ. Microcirculation in the ventricle of the dog and turtle. Circ Res. 1974;34:561-9.

23. Eng J, Lynch RM, Balaban RS. Nicotinamide adenine dinucleotide fluorescence spectroscopy and imaging of isolated cardiac myocytes. Biophys J. 1989;55:621-30.

24. An J, Camara AK, Rhodes SS, Riess ML, Stowe DF. Warm ischemic preconditioning improves mitochondrial redox balance during and after mild hypothermic ischemia in guinea pig isolated hearts. Am J Physiol Heart Circ Physiol. 2005;288:H2620-7.

25. Seal JB, Gewertz BL. Vascular dysfunction in ischemia-reperfusion injury. Ann Vasc Surg. 2005;19:572-84.

26. Mehlhorn U, Geissler HJ, Laine GA, Allen SJ. Myocardial fluid balance. Eur J Cardiothorac Surg. 2001;20:1220-30.

27. Menger MD, Pelikan S, Steiner D, Messmer K. Microvascular ischemia-reperfusion injury in striated muscle: significance of "reflow paradox." Am J Physiol. 1992;263:H1901-6.

28. Chamoun F, Burne M, O’Donnell M, Rabb H. Pathophysiologic role of selectins and their ligands in ischemia reperfusion injury. Front Biosci. 2000;5:E103-9.

29. Nigam A, Kopecky SL. Therapeutic potential of monoclonal antibodies in myocardial reperfusion injury. Am J Cardiovasc Drugs. 2002; 2:367-76.

30. Niessen HW, Krijnen PA, Visser CA, Meijer CJ, Hack CE. Intercellular adhesion molecule-1 in the heart. Ann N Y Acad Sci. 2002;973: 573-85.

31. Tillmanns H, Neumann FJ, Tiefenbacher C, Dorigo O, Parekh N, Waas W, et al. Activation of neutrophils in the microvasculature of the ischaemic and reperfused myocardium. Eur Heart J. 1993;14(Suppl I): $82-6$. 


\section{Online-Only References}

E1. Menger MD, Lehr HA. Scope and perspectives of intravital microscopybridge over from in vitro to in vivo. Immunol Today. 1993;14: 519-22.

E2. Wan MX, Schramm R, Klintman D, Welzenbach K, Weitz-Schmidt G, Thorlacius H. A statin-based inhibitor of lymphocyte function antigen-1 protects against ischemia/reperfusion-induced leukocyte adhesion in the colon. Br J Pharmacol. 2003;140:395-401.

E3. Schramm R, Yamauchi J, Vollmar B, Vajkoczy P, Menger MD. Blockade of in vivo VEGF-KDR/flk-1 signaling does not affect revascularization of freely transplanted pancreatic islets. Transplantation. 2003;75:239-42.

E4. Vollmar B, Janata J, Yamauchi JI, Menger MD. Attenuation of microvascular reperfusion injury in rat pancreas transplantation by L-arginine. Transplantation. 1999;67:950-5.
E5. Vollmar B, Glasz J, Menger MD, Messmer K. Leukocytes contribute to hepatic ischemia/reperfusion injury via intercellular adhesion molecule-1-mediated venular adherence. Surgery. 1995;117:195-200.

E6. Blanchard JM, Pollak R. Techniques for perfusion and storage of heterotopic heart transplants in mice. Microsurgery. 1985;6:169-74.

E7. Baran KW, Nguyen M, McKendall GR, Lambrew CT, Dykstra G, Palmeri ST, et al. Double-blind, randomized trial of an anti-CD18 antibody in conjunction with recombinant tissue plasminogen activator for acute myocardial infarction: limitation of myocardial infarction following thrombolysis in acute myocardial infarction (LIMIT AMI) study. Circulation. 2001;104:2778-83.

E8. Rusnak JM, Kopecky SL, Clements IP, Gibbons RJ, Holland AE, Peterman HS, et al. An anti-CD11/CD18 monoclonal antibody in patients with acute myocardial infarction having percutaneous transluminal coronary angioplasty (the FESTIVAL study). Am J Cardiol. 2001;88:482-7. 